Revista aSEPHallus de Orientação Lacaniana

Núcleo Sephora de Pesquisa sobre o Moderno e o Contemporâneo

ISSN $1809-709 \mathrm{X}$

\title{
L'Autre lacanien, une raison dans le réel $^{1}$
}

\section{Dany-Robert Dufour} Philosophe

Professeur retraité de I'Université Paris VIII

Auteur de $A$ Cidade perversa (Ed. Civilização Brasileira), O divino Mercado (Ed. Companhia de Freud), Os mistérios da trindade (Ed. Companhia de Freud), $A$ arte de reduzir as cabeças (Ed. Companhia de Freud), Le délire occidental (Éditions Les Liens qui libèrent) et $A$ existência de Deus comprovada por por um filósofo ateu

(Ed. Civilização Brasileira)

E-mail: dany.dufour@free.fr

Resumo: Não esperamos nossa época para percebermos a prematuração do homem. Existe todo um campo de estudos presente desde os primórdios da filosofia ocidental. Desde o século XX, os antropólogos e os biólogos falam da neotenia do homem. Se os períodos precedentes definiam espaços marcados pela distância entre o sujeito e aquele que o funda, a pós-modernidade é um espaço definido pela abolição da distância entre o sujeito e o Outro. Quando o Outro falta, podemos erigir com toda força um tipo de Outro que assegure absolutamente o sujeito contra todo risco de ausência.

Palavras-chave: neotenia; Outro; real; pós-modernidade.

\section{L'Autre lacanien, une raison dans le réel}

On n'a pas attendu notre époque pour s'apercevoir la prématuration de l'homme. Il existe tout un champ d'études présent depuis les débuts de la philosophie occidentale. Depuis le XXe siècle, les anthropologues et les biologistes parlent de la néoténie de l'homme. Si les périodes précédentes définissaient des espaces marqués par la distance du sujet à ce qui le fonde, la post-modernité est un espace défini par l'abolition de la distance entre le sujet et l'Autre. Lorsque l'Autre manque, on peut ériger à toute force une sorte d'Autre qui garantisse absolument le sujet contre tout risque d'absence.

Mots-clés: néoténie; Autre; réel; post-modernité.

\section{The lacanian Other, a reason in the real}

We do not wait for our time to realize the prematuration of man. There is a whole field of study present since the beginnings of western philosophy. Since the twentieth century, anthropologists and biologists have been talking about man's neoteny. If the preceding periods defined spaces marked by the distance between the subject and the one that founds him, postmodernity is a space defined by the abolition of the distance between the subject and the Other. When another is lacking, we can erect with all force a type of Other that absolutely assures the subject against all risk of absence.

Keywords: neoteny; Other; real; postmodernity. 


\section{L'Autre lacanien, une raison dans le réel}

\section{Dany-Robert Dufour}

Je remercie vivement les organisateurs de ce VI Simpósio do ISEPOL de m'avoir invité, notamment Tania Coelho dos Santos et Flávia Lana Garcia de Oliveira que j'ai rencontrée à Paris. Il faut cependant que vous sachiez que j'ai un gros défaut: je ne suis pas psychanalyste, mais philosophe. Pour ma défense, je dirai que je suis un philosophe un peu bizarre puisque je fréquente depuis longtemps la psychanalyse, non seulement ses auteurs, notamment Freud et Lacan, mais aussi ses praticiens, à quelque école qu'ils appartiennent. C'est ainsi, par exemple, qu'une belle amitié m'a liée au regretté Serge Leclaire à qui je dois beaucoup. Mais je ne suis pas ici pour vous raconter ma vie.

Si je suis ici, c'est parce qu'un livre que j'ai publié en France il y a quelques années vient d'être traduit aux éditions Civilizaçao Brasileira. Or, il se trouve que je fais, dans ce livre, référence à une notion que Freud et Lacan ont beaucoup utilisée, la notion de prématuration. Ce qui réfère au fait que l'homme est le seul des mammifères supérieurs qui naît notoirement inachevé à la naissance. Bref, il vient au monde trop tôt: mal fini, "bâclé" diraient certains, c'est-à-dire "ni fait ni à faire", comme on dit péjorativement en français. Voire même "salopé", comme le dénonçait avec force et désespoir Antonin Artaud.

Voici quelques preuves ou indices de cette prématuration spécifique de l'homme à la naissance: cloisons cardiaques non fermées, immaturité post-natale du système nerveux pyramidal, insuffisance des alvéoles pulmonaires, boîte crânienne non fermée (cf. la fontanelle), circonvolutions cérébrales à peine développées, absence de pouce postérieur opposable, absence de système pileux, absence de dentition de lait à la naissance - sans compter, chez les hommes, l'absence d'os pénien qui, dans certaines circonstances, peut faire cruellement défaut - n'est-ce pas chers amis. Bref, I'homme est un être à naissance prématurée, sujet à un très long maternage, incapable d'atteindre son développement germinal complet et cependant capable de se reproduire et de transmettre ses caractères de juvénilité, normalement transitoires chez les autres animaux.

On n'a pas attendu notre époque pour s'apercevoir de cette prématuration de l'homme. J'ai

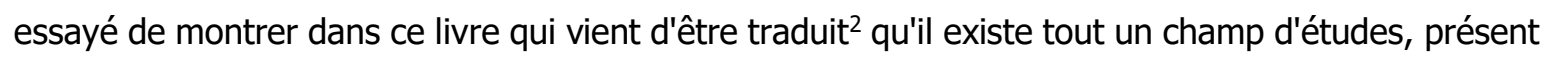
depuis les débuts de la philosophie occidentale, qui s'est penché sur cette question de l'inachèvement originaire de l'homme - je pense par exemple au Protagoras de Platon. Et cela s'est continué jusqu'au $X X^{e}$ siècle puisqu'alors les anthropologues et les biologistes ont parlé, pour évoquer cette prématuration, de la néoténie de l'homme ${ }^{3}$ et ceci dès 1926, depuis le célèbre article de l'anatomiste hollandais Louis Bolk.

C'est donc par là que l'aventure humaine commence, avec un être non advenu, incapable de se débrouiller seul - je l'appellerai donc le très-bas. Freud, pour sa part, avance ici le terme de Hilflosigkeit, desamparo en espagnol et en portugais, qui se rapporte à la détresse originaire de 
I'homme. On ne sait pas assez que ce concept scintille tout au long de la longue élaboration freudienne (la première occurrence apparaît dès 1895 et l'ultime en 1937). On trouve notamment dans le texte intitulé Inhibition, symptôme, angoisse publié en 1926, la même année que l'article inaugural de Bolk, cette notation ouvertement néoténique: "Parmi les facteurs qui participent à la causation des névroses (...), [il faut retenir] l'état de détresse et de dépendance [Hilflosigkeit] longuement prolongée du petit enfant d'homme. L'existence intra-utérine de l'homme apparaît face à celle de la plupart des animaux relativement raccourcie; l'enfant d'homme est jeté dans le monde plus inachevé qu'eux" (Freud, 1926/1993). Freud donc accorde une place tout à fait centrale à la néoténie puisqu'elle lui permet une construction tout à fait originale du fait névrotique. À la question traitée dans ce texte, "D'où vient la névrose?", Freud répond en effet en repérant trois facteurs essentiels parmi lesquels se trouve en premier lieu, le facteur biologique, l'état de détresse et de dépendance du petit de l'homme, explicitement lié à sa prématuration: "Ce facteur biologique instaure donc les premières situations de danger et crée le besoin d'être aimé, qui ne quittera plus l'être humain". La néoténie, avance en quelque sorte Freud, crée le besoin d'amour, lequel engendre la névrose.

Où Freud a-t-il été chercher ce terme? Je dois vous avouer ici mes penchants coupables pour les études théologiques. C'est ce qui m'a amené un jour à découvrir que ce terme de Hilflosigkeit venait tout simplement de la théologie de langue allemande, et plus précisément de Luther. Lequel emploie en effet beaucoup le terme Hilfe, l'"aide", en l'occurrence l'aide de dieu, nécessaire pour secourir I'homme, cet hilf-los (c'est-à-dire privé de hilfe), cet homme si bas. Si bas pour Luther que celui-ci a été dans un fameux sermon jusqu'à le comparer "au déchet tombé dans le monde par l'anus du diable"4 - ce qui nous emmène à l'exact opposé de l'homme comme roi de la création (Lacan, 1959-1960/1986). La néoténie, la Hilflosigkeit, engendre donc un besoin d'amour, lequel peut aller jusqu'au besoin d'amour de dieu.

Or, c'est là une idée que Lacan reprendra dès son entrée en psychanalyse, dans "le stade du miroir" de 1936 dont le texte original est, comme vous le savez, perdu, mais qu'on connaît plus ou moins par l'article de 1938 sur "la famille" paru dans l'Encyclopédie française, puis par la réécriture de ce texte en 1949. Lacan y fait explicitement de la néoténie de l'homme un pivot de sa démonstration (je cite la phrase-clef: "il ne faut pas hésiter à considérer l'homme comme un animal à naissance prématurée").

La thèse de Lacan sur le stade du miroir fait en effet une place de choix à la néoténie puisque c'est de cela qu'il part. l'inachèvement organique de l'homme, apprend-on, est suppléé par une épreuve décisive de nature psychique dans le procès de formation de l'individu. Cette épreuve, Lacan l'emprunte aux thèses néo-darwiniennes de Wallon (exposées dans Les origines du caractère chez l'enfantpublié en 1934). Elles font de la captation spéculaire et spectaculaire, où l'enfant se reconnaît et unifie son moi dans l'espace, le moment d'une épreuve qui commande l'accès à un ordre de coordination plus ample. La particularité de cette épreuve vient de ce qu'elle est aussi bien à entendre 
comme le dernier acte de maturation naturelle que comme le premier acte culturel qui précipite décisivement le sujet dans le monde humain. On doit en somme comprendre le stade du miroir comme le moment décisif où le manque de corps du néotène le voue à déployer sa vie dans une irrémédiable fiction. Le réel - le manque de corps - et l'imaginaire - être condamné à la fiction - sont en somme liés.

On pourrait balayer cette référence de Lacan à la néoténie comme anecdotique et la renvoyer à l'histoire ancienne. Or, près de quarante ans après le stade du miroir, où il avait coutume de placer son entrée en psychanalyse, Lacan, dans la dernière décade de son enseignement, en 1974, a persisté et signé pour dire que la néoténie de Bolk était fondamentale à son élaboration. Dans "La troisième", c'est-à-dire la $3^{\text {ème }}$ et dernière conférence donnée à Rome, Lacan déclare en effet, dans sa syntaxe si particulière:

Le rapport de l'homme, de ce qu'on appelle de ce nom, avec son corps, s'il y a quelque chose qui souligne bien qu'il est imaginaire, c'est la portée qu'y prend l'image et au départ, j'ai bien souligné ceci, c'est qu'il fallait pour ça quand même une raison dans le réel, et que la prématuration de Bolk - ce n'est pas de moi, c'est de Bolk, moi je n'ai jamais cherché à être original, j'ai cherché à être logicien - c'est qu'il n'y a que la prématuration qui l'explique, cette préférence de l'image qui vient de ce que qu'il anticipe sa maturation corporelle, avec tout ce que ça comporte, bien sûr, à savoir qu'il ne peut pas voir un de ses semblables sans penser que ce semblable prend sa place, donc naturellement qu'il le vomit. (Lacan, 2011, p. 22-23)

Quelques lignes plus bas, Lacan ajoute, en une notation lapidaire, une idée capitale puisqu'elle permet tout simplement de faire le lien entre la néoténie et l'Autre, grand A: "Ce qu'il y a de plus épatant, c'est que ça [la prématuration, l'anticipation de la maturation par l'image et la rivalité] a permis le glissement du commandement de Dieu". La remarque, sibylline, n'est guère développée, mais on y entend cependant que l'Autre, et même l'Autre en apothéose, Dieu lui-même, s'est introduit dans l'affaire humaine par le biais du réel de la néoténie!

Alors, cela m'a permis de conjecturer que le sujet, ce très-bas, ne trouve en général rien de mieux à faire, pour tenter de se sortir d'affaire, que de se soumettre à ce qu'il faut bien appeler le Très-haut. Je veux dire qu'il y aura remédiation si moi, être fini dans le temps et dans l'espace, mais si mal fini dans le réel, je parviens à supposer un être infini par rapport auquel je me mets en position de tout devoir. Or, supposer cet être, je le peux puisque je parle et que parler, c'est aussi fabuler. Rien donc ne m'empêche d'inventer dans le discours ce qui n'existe pas dans le réel, mais dont j'ai besoin pour vivre. Car, si je le suppose, Lui, le Très-Haut, celui que j'appelle le grand Sujet, alors je pourrai me "sous-poser" comme son sujet - c'est d'ailleurs exactement ce que veut dire le mot "sujet". Sujet, Subjectum, cela veut dire soumis, c'est-à-dire être "mis sous", c'est-à-dire "posé 
dessous". Il faut et il suffit donc que je conjecture un grand Sujet supposé tout savoir, tout pouvoir et tout voir pour que je trouve enfin ma place, comme sujet de cet être. Celui que j'appellerai le grand Sujet répond alors à cette définition d'Aristote: "Il est celui, disait-il dans la Métaphysique, dont tout le reste s'ordonne". En d'autres termes, I'homme est certes un "animal politique", comme le disait Aristote, mais est de surcroît un étrange animal, puisque non fini, qui, à la différence des autres animaux, doit se parachever ailleurs que dans la première nature, c'est-à-dire dans une seconde nature, généralement appelée culture. Une culture au centre de laquelle trône en quelque sorte un grand Sujet. Ce qui pourrait se reformuler ainsi: I'homme est un néotène qui tente de se soigner en devenant un animal théologico-politique, c'est-à-dire un "animal qui vénère", comme disait Nieztsche.

Cela veut dire que la survie de l'homme, animal néoténique, manquant comme tel de nature, passe par la création d'êtres de surnature, c'est-à-dire d'êtres de culture qui, bien que n'existant pas, se révèlent dotés d'une puissante efficacité symbolique.

Or, c'est très fatigant de créer des êtres de surnature. Il faut en effet mobiliser toutes les capacités de représentation sonore, visuelle et narrative afin de donner une allure, une figure, une voix, une histoire, une résidence à ce grand Sujet qu'à défaut de présence, il faut bien re-présenter, y compris sous la forme de l'irreprésentable.

Bref, l'art (récits, musique, arts plastiques...) sert à créer du grand Sujet. Mieux: l'art, c'est l'art de créer du grand Sujet. Autrement dit, ce qui manque dans la nature, on l'inventera donc la culture, grâce au langage, grâce aux récits, grâce à l'art, grâce aux mythes. Tel est donc le travail de la culture: il permet tout simplement la subjectivation. Dès que ce grand Sujet est en place, on obtient en effet la structure fondamentale de la subjectivation où l'on pose en dessous le petit sujet et en haut le grand Sujet. On peut donc dire que l'assomption subjective passe par la supposition de I'Autre, grand A.

Ce remède symbolique à la détresse réelle de l'homme relève bien sûr de ce que Platon appelait un pharmakon: un remède et un poison ${ }^{5}$. Bref, l'Autre, ce grand Sujet qui n'existe pas, est d'un grand secours... jusqu'à ce qu'il devienne extrêmement embarrassant.

La supposition de l'Autre est un remède parce qu'elle me sort de ma détresse originaire, et elle est un poison parce qu'elle m'aliène dans l'Autre. En d'autres termes, cette structure, comme le disait Freud, produit de la névrose. La névrose résulte en effet de la dette symbolique contractée à l'endroit de l'Autre. Ce qui nous amène à quelques considérations cliniques.

Cette structure produit de la névrose parce qu'elle m'incite à croire que, dans ce monde dans lequel je suis tombé, c'est l'Autre qui donne et que je lui dois tout. Il me fait notamment deux donations, l'une réelle (la vie) et l'autre symbolique (le langage), si exorbitantes l'une et l'autre que je ne pourrai jamais vraiment les rembourser. De sorte que je ne peux que culpabiliser.

Cette structure permet, me semble-t-il, de donner une forme nouvelle, dépassant la clinique individuelle, à ce que Freud avait appelé, dans un texte aussi bref que décisif, parmi ses tout derniers, 
la division subjective (la Spaltung) ${ }^{6}$. La division subjective, c'est ce qui fait de nous sommes des êtres clivés, ne se trouvant jamais vraiment, car, au moment même où l'on pourrait se trouver, on se perd dans l'Autre. C'est une question que Lacan a beaucoup travaillée dans le sens où il a systématiquement exploré les effets de refoulement originaire produits par la confrontation du petit sujet au grand Sujet, c'est-à-dire telle ou telle figure de l'Autre.

Je crois cependant qu'on se doit de reprendre aujourd'hui la question. Pourquoi? Parce que Lacan a été l'homme de son temps, c'est-à-dire l'homme des temps structuralistes qui ont privilégié la synchronie et révélé qu'il existait un Autre de structure. Or, il est peut-être temps maintenant de passer à la diachronie, c'est-à-dire à la question de la variation historique des figures de l'Autre. C'est la condition pour passer du clinique au politique. Je veux dire que l'Autre, cet être de fiction qui n'existe pas, ne peut véritablement être perçu dans son efficace que si l'on saisit, identifie et répertorie, disons, ses avatars différents. C'est pourquoi je me suis donné comme programme de m'engager dans un repérage historique des figures de l'Autre. Ce qui m'a conduit à penser que I'histoire humaine se caractérise par un renouvellement permanent de la figure de l'Autre.

Et, de fait, lorsqu'on se penche sur l'histoire, on trouve cet Autre dans tous les mondes possibles construits par l'homme. Soit sous la forme du Totem, par exemple, par quoi un groupe d'hommes se désignent un mâle dominant (le Totem, qui a beaucoup intéressé Feud, fait remonter les filiations à une puissance de la première nature, un faucon, un jaguar ou un autre "vrai" animal, de sorte que l'anima du groupe rencontre l'Animal...). Soit sous la forme d'esprits qui habitent, voire qui hantent, les lieux où résident les hommes. Soit sous la forme de dieux immanents au monde qui, comme les dieux grecs de la Physis par exemple, interviennent sans cesse dans les affaires de l'homme. Soit sous la forme d'un Dieu transcendant comme dans les monothéismes qui figure un Père absolu, éternel. Soit même sous la forme de religions politiques, comme dans l'absolutisme royal. Mais ce dernier grand Sujet était probablement trop grand puisqu'il a fallu le raccourcir, c'està-dire le couper, c'est-à-dire lui couper la tête. Cela s'appelle la révolution française qui n'a décapité le Roi, grand Sujet de l'Ancien Régime, que pour faire apparaître un nouveau grand Sujet, le Peuple. N'oublions pas enfin les religions politiques que le $X X^{e}$ siècle a connues, comme le stalinisme qui présentait le Prolétariat comme le sauveur et comme le nazisme qui célébrait une soi-disant race supérieure. Tous ces Autres ont permis la fonction symbolique dans la mesure où ils ont donné un point d'appui au sujet pour que ses discours reposent sur un fondement. Mais bien sûr, certains ont été plus pérennes que d'autres. 
Or, vous savez qu'il y a une trentaine d'années, en 1979 exactement, et c'est là où ma motion philosophique réapparaît, le philosophe J. F. Lyotard a fait l'hypothèse de l'apparition d'une cassure dans la modernité. Dans un remarquable essai intitulé La condition post-moderne, paru une année avant la prise du pouvoir par des néo-libéraux dans deux des plus importants pays du monde, Thatcher en Angleterre et Reagan aux États-Unis, Lyotard notait que nous étions en train de sortir de la modernité et d'entrer dans une époque "post-moderne" - Lyotard entendait par là l'épuisement et la disparition des grands récits de fondation. Dans cet audacieux livre, Lyotard annonçait - sans trop sembler s'en réjouir, ni d'ailleurs en être accablé - la nouvelle donne: nous étions en train de sortir des grands récits théologico-politiques, tant anciens (ceux des monothéismes) que modernes (les récits de l'émancipation individuelle par l'accès à la raison critique - Kant - ou ceux de l'émancipation sociétale - le marxisme par exemple). Or, la chute des grands récits, c'est aussi la chute des figures de l'Autre que ces récits soutenaient. Voici donc, dans la post-modernité, notre structure lourdement atteinte. Ce qui ne peut qu'entraîner une transformation assez radicale de la condition subjective. Je veux dire que le fait d'être sujet se présente aujourd'hui, dans nos démocraties de marché, sous une modalité assez sensiblement différente de ce que cela fut pour les générations précédentes. En bref, je n'hésiterai pas à conjecturer que le sujet qui se présente aujourd'hui n'est globalement plus le même que celui qui s'y présentait il y a encore une génération. Je dis donc que la condition subjective est soumise, elle aussi, à la variation historique et que nous venons probablement de franchir à cet égard un cap important, ce qui entraine un profond remaniement des grandes institutions, comme la famille, l'école, sans oublier les institutions de soins.

C'est pourquoi, après avoir parlé de décliner les figures de l'Autre (comme on décline une identité), je voudrais maintenant parler du déclin de l'Autre - en somme, après s'être décliné, l'Autre s'incline. C'est bien sûr intéressant que les idoles s'effondrent comme le souhaitait Nietzsche, mais il ne faudrait pas ignorer les sérieux problèmes que cela pose. Car la post-modernité n'a plus de figures présentables de l'Autre à proposer. Si les périodes précédentes définissaient des espaces marqués par la distance du sujet à ce qui le fonde, alors la post-modernité est un espace défini par l'abolition de la distance entre le sujet et l'Autre.

En fait d'Autre, nous avons désormais le Marché. Le Marché qui est souvent présenté comme le remède à tous les maux. Sauf que le Marché ne fonctionne plus vraiment comme un grand récit, mais plutôt comme une addition à l'infini de petits récits égotiques. C'est pourquoi le Marché ne vaut nullement comme nouvel Autre, dans la mesure où loin de prendre en charge la question de l'origine, il confronte chacun aux affres (qui ne vont certainement pas sans nouvelles jouissances) de l'autofondation. C'est là où se repère la limite fondamentale de l'économie de marché dans sa prétention à prendre en charge l'ensemble du lien personnel et du lien social: ce n'est pas une économie 
générale, pas une économie symbolique, mais seulement une "économie économique". Elle joue certes dans le registre de l'économie libidinale dans la mesure où elle permet toujours de présenter un objet manufacturé, un service marchand ou un fantasme sur mesure produit par les industries culturelles, supposé venir combler tout appétence, mais elle échoue à fonctionner comme ce que Bataille appelait une économie générale dans la mesure où elle laisse le sujet face à lui-même pour ce qu'il en est de sa fondation.

Cette distance interne du sujet à lui-même se découvre inhérente au sujet post-moderne et modifie sensiblement le diagnostic de Freud sur le sujet moderne, porté à la névrose. C'est, je crois, vers une condition subjective définie par un état-limite entre névrose et psychose que se définit désormais le sujet post-moderne, de plus en plus pris entre mélancolie latente (la fameuse dépression), emmêlement intérieur, impossibilité de parler en première personne, illusion de toutepuissance et fuite en avant dans des faux self, dans des personnalités d'emprunt, offertes à profusion par le marché.

L'un des effets directs de cette nouvelle donne est certains sujets se sont alors sentis comme orphelins de l'Autre et ont cherché, comme ils le peuvaient, à obvier au défaut de l'Autre.

Par exemple, lorsque l'Autre manque, on peut tenter de réinscrire l'Autre dans l'ordre non plus du désir, mais du besoin. La marchandise peut jouer ce rôle. On voit ainsi se développer de véritables addictions à la marchandise. À toutes les marchandises, mais à une en particulier, celle qui bénéficie du terrible privilège d'être illicite, rare et chère, donc extrêmement tentante entre toutes les marchandises, je veux parler bien sûr de la drogue. Avec la drogue, on réinscrit l'Autre dans l'ordre non plus du désir, mais du besoin. C'est ce qui est à l'œuvre dans la toxicomanie où l'enjeu n'est plus de faire de la difficulté d'exister une quête symbolique où ce qui vient combler l'imperfection usuelle de l'Autre doit être savamment construit et exprimé, notamment par la création artistique (poèmes, danse, chant, musique, peinture...). Dans la toxicomanie, cette laborieuse quête est transformée en une simple dépendance à l'égard d'un Autre sorti du champ du désir et réinscrit en quelque sorte dans le réel du besoin. Au moins, saura-t-on ainsi où est et ce qu'il en est de l'Autre dont on manque : rien d'autre qu'un produit chimique aussi addictif que possible que l'on pourra se procurer à condition qu'on en devienne l'esclave. On connaît les effets dévastateurs de l'usage intensif, je ne parle pas de l'usage occasionnel, de drogues vis-à-vis.

Deuxième exemple, lorsque l'Autre manque, on peut se passer de l'Autre à la condition de se trouver soi-même investi, ne serait-ce que pendant quelques minutes, des signes de la toutepuissance qui le caractérisaient. Dans les cas extrêmes, on pourra même s'octroyer droit de vie et de mort sur ses semblables et que l'on se dotera éventuellement de pouvoirs magiques, d'autant plus facilement que le prestige social des techno-sciences, renforçant constamment l'environnement prothétique, ne va pas sans exalter les sentiments de toute puissance du sujet (caméra GoPro, armes sophistiquées, technologies numériques de l'image, du son, de l'internet qui permettent à n'importe qui de s'adresser au monde entier...).. Les actes de violence les plus crus peuvent alors déferler sans 
aucune retenue. On ne compte plus, depuis les débuts de la postmodernité, les passages à l'acte violent qui défraient la chronique. Depuis le crime du caporal Lortie dans l'Assemblée nationale du Québec en 1984, jusqu'à la tuerie de l'école primaire de Sandy Hook aux États-Unis, le nombre de fusillades et de tueries augmente d'année en année (330 fusillades de masse en 2015 aux Etats-Unis se soldant par quelques près de 14.000 meurtres) ${ }^{7}$.

Troisième exemple, lorsque l'Autre manque, on peut ériger à toute force une sorte d'Autre qui garantisse absolument le sujet contre tout risque d'absence. C'est ce qu'on voit à l'œuvre dans les multiples sectes qui fleurissent dans les sociétés post-modernes - un petit groupe s'assemble, brandit l'effigie d'un gourou ou du nouveau maître absolu ou d'un dieu archaïque vengeur et farouche. Je n'insiste pas, mais je suis bien tenté de dire qu'à l'époque de la mondialisation, cet affrontement peut prendre, avec les fondamentalismes, une tournure globale: Jihad contre Marché total.

Et je dirai pour terminer que rien n'interdit à ces orphelins de l'Autre de combiner ces différentes tendances. On trouve de plus en plus de sujets qui sont à la fois addictés, sujets à la toute-puissance et enclins au radicalisme fondamentaliste.

Toutes ces tendances sont analysables comme des réactions à la perte des pères et des repères provoquée par la culture post-moderne. Mais l'histoire, contrairement au souhait des adulateurs du Marché, ne s'arrête jamais. Ces formes laissent présager l'apparition prochaine d'un autre régime clinico-politique, non plus ultra-démocratique, mais ultra-autoritaire, renouant avec les anciennes religions politiques fascisantes dont on croyait (à tort) s'être libérés depuis des décennies. En d'autres termes, la mondialisation néo-libérale, qui a dissout les identités nationales, les patriarcats et les patries, provoque un choc en retour et la réapparition d'idéologies identitaires autoritaires - cela monte partout dans ce qu'on appelle au Brésil le "premier monde", c'est-à-dire en Europe et aux États-Unis avec le "trumpisme". Ce qui pourrait augurer d'un prochain grand renversement car alors l'ethnos, plutôt que le démos, pour la première fois depuis 70 ans (la fin de la seconde guerre mondiale), requalifierait en quelque sorte l'Autre.

On pourrait dire que tout cela - ces formes toujours renouvelées que l'Autre ne cesse de prendre au fil de l'histoire - résulte de l'incessant et incertain traitement narratif de la néoténie humaine. Ce qui se traduit par un symbolique lesté en son centre d'une forme imaginaire surdéterminante sujette à de brusques renversements, exhibant souvent, contre quelques carottes, beaucoup de bâtons.

Mais, pendant que l'humanité continue à se raconter des histoires (évidemment vaines), d'aucuns se mettent à penser qu'il pourrait exister non seulement un traitement symbolique, mais aussi et surtout un traitement rée/ du problème dont souffre constitutivement l'humain. On sort là 
de la post-modernité pour entrer (en parfaits aveugles) dans une ère nouvelle : celle de la posthumanité. Il s'agira alors de tenter de réparer, grâce aux techno-sciences, l'erreur humaine, en vue de refaire en mieux, du moins l'espère-t-on, ce néotène venu du fond des âges. C'est là toute la thématique aujourd'hui soutenue par le posthumanisme. Celle de I'homme augmenté où I'homme devient un pur et simple OGM, un organisme génétiquement modifiable.

J'ai épuisé mon temps de parole, je me permets donc de renvoyer donc à la fin de mon livre où j'examine ce qu'il en est de cette nouvelle forme de folie humaine où I'homme, soutenu par une passion faustienne qui n'est plus seulement incantatoire, mais opérante, pourrait bien, grâce aux techno-sciences, changer le réel de l'homme.

J'avoue pour ma part mon très grand embarras à choisir entre les traitements narratifs actuellement disponibles (en gros, le Marché, le Jihadisme ou le Trumpisme, soit trois formes du pire) et un traitement réel prochain qui propose en quelque sorte de soigner la maladie en faisant purement et simplement disparaître le malade.

Mais peut-être que mes amis psychanalystes sauraient suggérer un traitement acceptable. Je vous écoute donc et je vous remercie.

\section{Notes:}

${ }^{1}$ Texte presenté par l'auteur au VI Simpósio do ISEPOL, le 30/09/2016.

${ }^{2}$ Dufour, D-R. On achève bien les hommes, Denoël, Paris 2005, traduit sous le titre $A$ existência de Deus comprovada por um filósofo ateu, Rio de Janeiro, 2016.

3 Je fais référence aux recherches qui se sont développées (sous le nom d'"anthropologie philosophique") à la suite des travaux de Louis Bolk ("Le problème de la genèse humaine" (Das Problem der Menschwerdung, 1926. Elles passent par des auteurs de langue allemande comme Adolf Portmann et Arnold Gehlen et se sont prolongées par les recherches décisives de l'américain Stephen Jay Gould. Dont sa théorie de la néoténie est développée dans Ontogeny and Phylogeny, par Harvard University Press, en 1977.

${ }^{4}$ Cette forte proposition concernant l'homme n'a pas échappé à Lacan puisqu'il la cite dans son séminaire L'éthique (dans la séance du 13 Janvier 1960). Ce qui tend à montrer que, si l'on veut comprendre quelque chose au symbolique, il faut partir du... diabolique.

${ }^{5}$ Je renvoie bien sûr à la lecture du Phèdre de Platon par Jacques Derrida dans La dissémination, "La pharmacie de Platon".

${ }^{6}$ Le terme allemand Spaltung est la traduction par Freud d'une expression fréquente dans la psychiatrie française, celle de "dissociation" référant entre autre à ce que Pierre Janet appelait la "double conscience". On le rend aussi en français par le terme de "clivage".

${ }^{7}$ Le site américain Gun Violence Archive (GVA), fondé en 2013, après la tuerie de l'école de Sandy Hook, a dénombré en 2015 aux USA 52666 incidents impliquant des armes à feu (10\% de plus que 
l'année précédente), dont 330 fusillades de masse, se soldant au total par 13350 meurtres et 26940 blessés.

\section{Références Bibliographiques}

Derrida, J. (1972). La pharmacie de Platon. La dissémination. Paris: Seuil.

Dufour, D.-R. (2005). $n$ achève bien les hommes. Paris: Denoël.

Freud, S. (1993). Inhibition, symptôme et angoisse. Paris: PUF (Oeuvre originale publiée en 1926).

Freud, S. (1938). La scission du moi dans le processus de defense. Recuperé de http://www.psychanalyse.lu/articles/FreudScission.htm.

Gantheret, F. \& Lapassade, G. (1961, abr.). Le problème de la genèse humaine. Revue française de Psychanalyse, 243-279.

Gould, S. J. (1977). Ontogeny and Phylogeny. Harvard University Press.

Lacan, J. (1975). La Troisième. Lettres de l'École Freudienne, 1(16), 177-203.

Lacan, J. (1986). Le séminaire, livre 7: l'éthique de la psychanalyse. Paris: Seuil (Oeuvre originale publiée en 1959-1960).

Citacão/Citation: Dufour, D.-R. (mai. a out. 2016). L'Autre lacanien, une raison dans le réel. Revista aSEPHallus de Orientação Lacaniana, 11(22), 31-41. Disponível em www.isepol.com/asephallus. doi: 10.17852/1809-709x.2019v11n22p31-41.

Editor do artigo: Tania Coelho dos Santos.

Recebido/Received: 01/06/2016 / 06/01/2016.

Aceito/Accepted: 05/06/2016 / 06/05/2016.

Copyright: (C) 2013 Associação Núcleo Sephora de Pesquisa sobre o moderno e o contemporâneo. Este é um artigo de livre acesso, que permite uso irrestrito, distribuição e reprodução em qualquer meio, desde que o autor e a fonte sejam citados/This is an open-access article, which permites unrestricted use, distribution, and reproduction in any medium, provided the author and source are credited. 